\title{
How Well Do Local Algorithms Solve Semidefinite Programs?*
}

\author{
Zhou Fan ${ }^{\dagger}$ \\ Stanford University \\ Department of Statistics \\ USA \\ zhoufan@stanford.edu
}

\begin{abstract}
Several probabilistic models from high-dimensional statistics and machine learning reveal an intriguing -and yet poorly understooddichotomy. Either simple local algorithms succeed in estimating the object of interest, or even sophisticated semi-definite programming (SDP) relaxations fail. In order to explore this phenomenon, we study a classical SDP relaxation of the minimum graph bisection problem, when applied to Erdős-Rényi random graphs with bounded average degree $d>1$, and obtain several types of results. First, we use a dual witness construction (using the so-called non-backtracking matrix of the graph) to upper bound the SDP value. Second, we prove that a simple local algorithm approximately solves the SDP to within a factor $2 d^{2} /\left(2 d^{2}+d-1\right)$ of the upper bound. In particular, the local algorithm is at most $8 / 9$ suboptimal, and $1+O(1 / d)$ suboptimal for large degree.

We then analyze a more sophisticated local algorithm, which aggregates information according to the harmonic measure on the limiting Galton-Watson (GW) tree. The resulting lower bound is expressed in terms of the conductance of the GW tree and matches surprisingly well the empirically determined SDP values on largescale Erdős-Rényi graphs.

We finally consider the planted partition model. In this case, purely local algorithms are known to fail, but they do succeed if a small amount of side information is available. Our results imply quantitative bounds on the threshold for partial recovery using SDP in this model.
\end{abstract}

\section{CCS CONCEPTS}

- Mathematics of computing $\rightarrow$ Random graphs; Graph algorithms; • Theory of computation $\rightarrow$ Semidefinite programming;

\footnotetext{
${ }^{*}$ A full version of this paper is available at http://arxiv.org/abs/1610.05350 ${ }^{\dagger}$ Partially supported by a Hertz Foundation fellowship and an NDSEG Fellowship (DoD AFOSR 32 CFR 168a).
}

${ }^{\ddagger}$ Partially supported by NSF grants CCF-1319979 and DMS- 1613091 .

Permission to make digital or hard copies of all or part of this work for personal or classroom use is granted without fee provided that copies are not made or distributed for profit or commercial advantage and that copies bear this notice and the full citation on the first page. Copyrights for components of this work owned by others than ACM must be honored. Abstracting with credit is permitted. To copy otherwise, or republish, to post on servers or to redistribute to lists, requires prior specific permission and/or a fee. Request permissions from permissions@acm.org.

STOC'17, June 2017, Montreal, Canada

(C) 2017 Association for Computing Machinery.

ACM ISBN 978-1-4503-4528-6/17/06...\$15.00

https://doi.org/10.1145/3055399.3055451

\author{
Andrea Montanari ${ }^{\ddagger}$ \\ Stanford University \\ Department of Electrical Engineering and Department of \\ Statistics \\ USA \\ montanari@stanford.edu
}

\section{KEYWORDS}

Local algorithm, semidefinite program relaxation, non-backtracking spectrum, community detection

ACM Reference format:

Zhou Fan and Andrea Montanari. 2017. How Well Do Local Algorithms Solve Semidefinite Programs? In Proceedings of 49th Annual ACM SIGACT Symposium on the Theory of Computing, Montreal, Canada, Fune 2017 (STOC'17), 11 pages.

https://doi.org/10.1145/3055399.3055451

\section{INTRODUCTION}

Semi-definite programming (SDP) relaxations are among the most powerful tools available to the algorithm designer. However, while efficient specialized solvers exist for several important applications [BM03, WS08, NN13], generic SDP algorithms are not well suited for large-scale problems. At the other end of the spectrum, local algorithms attempt to solve graph-structured problems by taking, at each vertex of the graph, a decision that is only based on a bounded-radius neighborhood of that vertex [Suo13]. As such, they can be implemented in linear time, or constant time on a distributed platform. On the flip side, their power is obviously limited.

Given these fundamental differences, it is surprising that these two classes of algorithms behave similarly on a number of probabilistic models arising from statistics and machine learning. Let us briefly review two well-studied examples of this phenomenon.

In the (generalized) hidden clique problem, a random graph $G$ over $n$ vertices is generated as follows: A subset $S$ of $k$ vertices is chosen uniformly at random among all $\left(\begin{array}{l}n \\ k\end{array}\right)$ sets of that size. Conditional on $S$, any two vertices $i, j$ are connected by an edge independently with probability $p$ if $\{i, j\} \subseteq S$ and probability $q<p$ otherwise. Given a single realization of this random graph $G$, we are requested to find the set $S$. (The original formulation [Jer92] of the problem uses $p=1, q=1 / 2$ but it is useful to consider the case of general $p$, q.)

SDP relaxations for the hidden clique problem have been studied in a number of papers, beginning with the seminal work of Feige and Krauthgamer [FK00, AV11, MPW15, DM15b, BHK $\left.{ }^{+} 16\right]$. Remarkably, even the most powerful among these relaxations, constructed through the sum-of-squares (SOS) hierarchy, fail unless $k \gtrsim \sqrt{n}\left[\mathrm{BHK}^{+} 16\right]$, while exhaustive search succeeds with high probability as soon as $k \geq C_{0} \log n$ for $C_{0}=C_{0}(p, q)$ a constant. Note that the standard definition of local algorithms is not useful for this problem, since the underlying graph is dense and hence the whole graph is within a bounded distance of any vertex. However, 
[Mon15] studied the sparse regime $p, q=\Theta(1 / n)$ and characterized the behavior of an optimal local algorithm that is related to the 'belief propagation' heuristic in graphical models. Belief propagation attempts to compute in an iterative fashion local posterior probabilities and can be applied to dense graphs as well (i.e. for $p, q=\Theta(1))$. Belief propagation was analyzed on dense graphs in [DM15a] and proven to succeed if and only if $k \geq C_{1} \sqrt{n}$. Summarizing, the full power of the SOS hierarchy, despite having a much larger computational burden, does not qualitatively improve upon the performance of simple local heuristics.

As a second example, we consider the two-groups symmetric stochastic block model (also known as the planted partition problem) that has attracted considerable attention in recent years as a model for community detection in networks [DKMZ11, $\mathrm{KMM}^{+} 13$, MNS13, Mas14, BLM15, GV15]. A random graph $G$ over $n$ vertices is generated by partitioning the vertex set into two subsets ${ }^{1} S_{+} \cup S_{-}$ of size $n / 2$ uniformly at random. Conditional on this partition, any two vertices $i, j$ are connected by an edge independently with probability $a / n$ if $\{i, j\} \subseteq S_{+}$or $\{i, j\} \subseteq S_{-}$(the two vertices are on the same side of the partition), and with probability $b / n$ otherwise (the two vertices are on different sides). Given a single realization of the random graph $G$, we are requested to identify the partition.

While several 'success' criteria have been studied for this model, for the sake of simplicity we will focus on weak recovery (also referred to as 'detection' or 'partial recovery'). Namely, we want to attribute $\{+,-\}$ labels to the vertices so that, with high probability, at least $(1 / 2+\varepsilon) n$ vertices are labeled correctly (up to a global sign flip that cannot be identified). It was conjectured in [DKMZ11] that this is possible if and only if $\lambda>1$, where $\lambda \equiv(a-b) / \sqrt{2(a+b)}$ is an effective 'signal-to-noise ratio' parameter. This conjecture followed from the heuristic analysis of a local algorithm based once again- on belief propagation. The conjecture was subsequently proven in [MNS12, MNS13, Mas14] through the analysis of carefully constructed spectral algorithms. While these algorithms are, strictly speaking, not local, they are related to the linearization of belief propagation around a 'non-informative fixed point'.

Convex optimization approaches for this problem are based on the classical SDP relaxation of the minimum-bisection problem. Denoting by $\boldsymbol{A}=\boldsymbol{A}_{G}$ the adjacency matrix of $G$, the minimum bisection problem is written as

$$
\begin{array}{ll}
\operatorname{maximize} & \langle\boldsymbol{\sigma}, \boldsymbol{A} \boldsymbol{\sigma}\rangle, \\
\text { subject to } & \boldsymbol{\sigma} \in\{+1,-1\}^{n},\langle\boldsymbol{\sigma}, \mathbf{1}\rangle=0 .
\end{array}
$$

The following SDP relaxes the above problem, where $d=(a+b) / 2$ is the average degree:

$$
\begin{array}{ll}
\text { maximize } & \left\langle A-\frac{d}{n} \mathbf{1 1}{ }^{\top}, X\right\rangle, \\
\text { subject to } & X \geq 0, \quad X_{i i}=1 \quad \forall i .
\end{array}
$$

(Here, the term $-(d / n) \mathbf{1 1}{ }^{\top}$ can be thought of as a relaxation of the hard constraint $\langle\boldsymbol{\sigma}, \mathbf{1}\rangle=0$.) This SDP relaxation has a weak recovery threshold $\lambda^{\text {sDP }}$ that appears to be very close to the ideal one $\lambda=1$. Namely, Guédon and Vershynin [GV15] proved $\lambda^{\mathrm{SDP}} \leq C$ for $C$ a universal constant, while [MS16] established $\lambda^{\mathrm{SDP}}=1+o_{d}(1)$ for large average degree $d$.

\footnotetext{
${ }^{1}$ To avoid notational nuisances, we assume $n$ even.
}

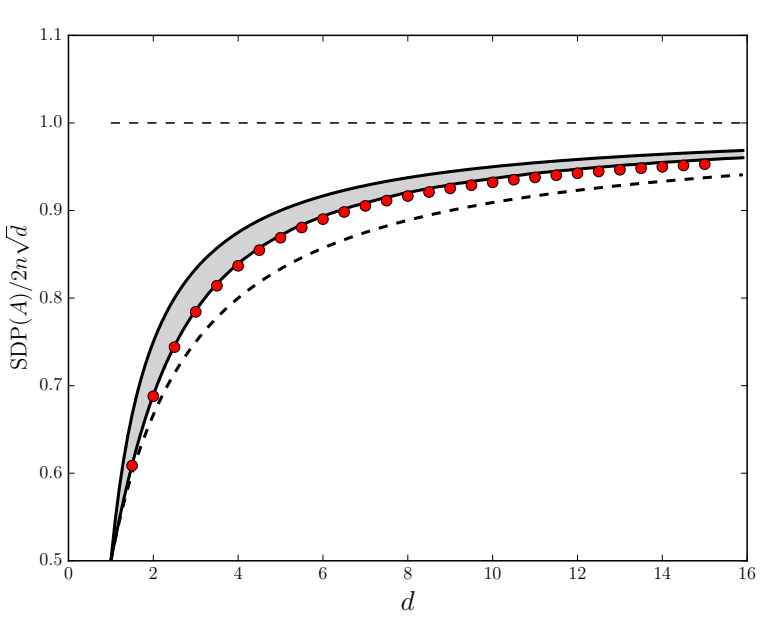

Figure 1: Typical value $\operatorname{SDP}\left(A_{G}\right)$ of the min-bisection SDP for large Erdős-Rényi random graphs with average degree $d$, normalized by the large degree formula $2 n \sqrt{d}$. Circles: numerical simulations with graphs of size $n=10^{6}$. Solid lines: Upper bound from Theorem 2.2 and local algorithm lower bound (evaluated numerically) from Theorem 2.4. Lower dashed line: Explicit local-algorithm lower bound from Theorem 2.2. (The small inconsistency between numerical SDP values and the lower bound at large $d$ is due to non-asymptotic effects that appear to vanish as $n \rightarrow \infty$.)

Summarizing, also for the planted partition problem local algorithms (belief propagation) and SDP relaxations behave in strikingly similar ways ${ }^{2}$. In addition to the above rigorous results, numerical evidence suggests that the two thresholds are very close for all degrees $d$, and that the reconstruction accuracy above these thresholds is also very similar [JMRT16].

The conjectural picture emerging from these and similar examples can be described as follows. For statistical inference problems on sparse random graphs, SDP relaxations are no more powerful than local algorithms (eventually supplemented with a small amount of side information to break symmetries). On the other hand, any information that is genuinely non-local is not exploited even by sophisticated SDP hierarchies. Of course, formalizing this picture is of utmost practical interest, since it would entail a dramatic simplification of algorithmic options.

With this general picture in mind, it is natural to ask: Can semidefinite programs be (approximately) solved by local algorithms for a large class of random graph models? A positive answer to this question would clarify the equivalence between local algorithms and SDP relaxations.

\footnotetext{
${ }^{2} \mathrm{An}$ important remark is that strictly local algorithms are ineffective in the planted partition problem. This peculiarity is related to the symmetry of the model, and can be resolved in several ways, for instance by an oracle that reveals an arbitrarily small fraction of the true vertex labels, or running belief propagation a logarithmic (rather than constant) number of iterations. We refer to Section 2.3 for further discussion of this point.
} 
Here, we address this problem by considering the semidefinite program (3), for two simple graph models, the Erdős-Rényi random graph with average degree $d, G \sim \mathrm{G}(n, d / n)$, and the two-groups symmetric stochastic block model, $G \sim \mathrm{G}(n, a / n, b / n)$. We establish the following results (denoting by $\operatorname{SDP}\left(\boldsymbol{A}_{G}\right)$ the value of (3)).

Approximation ratio of local algorithms. We prove that there exists a simple local algorithm that approximates $\operatorname{SDP}\left(A_{G}\right)$ (when $G \sim \mathrm{G}(n, d / n)$ ) within a factor $2 d^{2} /\left(2 d^{2}+d-1\right)$, asymptotically for large $n$. In particular, the local algorithm is at most a factor $8 / 9$ suboptimal, and $1+O(1 / d)$ suboptimal for large degree.

Note that $\operatorname{SDP}\left(\boldsymbol{A}_{G}\right)$ concentrates tightly around its expected value. When we write that an algorithm approximates $\operatorname{SDP}\left(\boldsymbol{A}_{G}\right)$, we mean that it returns a feasible solution whose value satisfies the claimed approximation guarantee.

Typical SDP value. Our proof provides upper and lower bounds on $\operatorname{SDP}\left(A_{G}\right)$ for $G \sim \mathrm{G}(n, d / n)$, implying in particular that $\operatorname{SDP}\left(A_{G}\right) / n=2 \sqrt{d}(1-\Theta(1 / d))+o_{n}(1)$ where the term $\Theta(1 / d)$ has explicit upper and lower bounds. While the lower bound is based on the analysis of a local algorithm, the upper bound follows from a dual witness construction, which is of independent interest. We analyze the dual witness construction using a generalization of the Ihara-Bass identity and a centered variant of the non-backtracking matrix of the graph.

Our upper and lower bounds are plotted in Fig. 1 together with the results of numerical simulations.

A local algorithm based on harmonic measures. The simple local algorithm above uses randomness available at each vertex of $G$ and aggregates it uniformly within a neighborhood of each vertex. We analyze a different local algorithm that aggregates information in proportion to the harmonic measure of each vertex. We characterize the value achieved by this algorithm in the large $n$ limit in terms of the conductance of a random GaltonWatson tree. Numerical data (obtained by evaluating this value and also solving the SDP (3) on large random graphs), as well as a large- $d$ asymptotic expansion, suggest that this lower bound is very accurate, cf. Fig. 1.

SDP detection threshold for the stochastic block model. We then turn to the weak recovery problem in the two-group symmetric stochastic block model $G \sim \mathrm{G}(n, a / n, b / n)$. As above, it is more convenient to parametrize this model by the average degree $d=(a+b) / 2$ and the signal-to-noise ratio $\lambda=$ $(a-b) / \sqrt{2(a+b)}$. It was known from [GV15] that the threshold for SDP to achieve weak recovery is $\lambda^{\mathrm{SDP}}(d) \leq 10^{4}$, and in [MS16] that $\lambda^{\mathrm{SDP}}(d) \leq 1+o_{d}(1)$ for large degree. Our results provide more precise information, implying in particular $\lambda^{\mathrm{SDP}}(d) \leq \min \left(2-d^{-1}, 1+C d^{-1 / 4}\right)$ for $C$ a universal constant.

Let us emphasize that our work leads to a number of interesting open questions. First of all, the exact asymptotic value of $\operatorname{SDP}\left(A_{G}\right)$ for Erdős-Rényi random graphs of fixed average degree $d>1$ is currently unknown. Second, it would be important to extend the present analysis to other random graph models, and to understand how general is the finding that local algorithms can approximately solve the SDP (3). Finally, we study here a particularly simple SDP relaxation (corresponding to the second level of the SOS hierarchy) and it would be interesting to understand to what extent the present picture extends to more sophisticated relaxations.

\section{MAIN RESULTS}

In this section we recall the notion of local algorithms, as specialized to solving the problem (3). We then state formally our main results. For general background on local algorithms, we refer to [HLS14, GS14, Lyo14]: this line of work is briefly discussed in Section 3.

Note that the application of local algorithms to solve SDPs is not entirely obvious, since local algorithms are normally defined to return a quantity for each vertex in $G$, instead of a matrix $X$ whose rows and columns are indexed by those vertices. Our point of view will be that a local algorithm can solve the SDP (3) by returning, for each vertex $i$, a random variable $\xi_{i}$, and the SDP solution $X$ associated to this local algorithm is the covariance matrix of $\xi=\left(\xi_{1}, \ldots, \xi_{n}\right)$ with respect to the randomness of the algorithm execution. A good approximation of this solution $X$ can be obtained by repeatedly sampling the vector $\xi \in \mathbb{R}^{n}$ (i.e. by repeatedly running the algorithm with independent randomness), and taking the empirical covariance of the samples. Standard concentration of measure arguments imply that a small number of samples (logarithmic in $n)$ are sufficient to obtain a $(1-\varepsilon)$ approximation of the value achieved by $X$.

Formally, let $\mathcal{G}$ be the space of (finite or) locally finite rooted graphs, i.e. of pairs $(G$,$) where G=(V, E)$ is a locally finite graph and $\in V$ is a distinguished root vertex. We denote by $\mathcal{G}^{*}$ the space of tuples $(G,, z)$ where $(G,) \in \mathcal{G}$ and $z: V \rightarrow \mathbb{R}$ associates a realvalued mark to each vertex of $G$. Given a graph $G=(V, E)$ and a vertex $i \in V$, we denote by $\mathrm{B}_{\ell}(i ; G)$ the subgraph induced by vertices $j$ whose graph distance from $i$ is at most $\ell$, rooted at $i$. If $G$ carries marks $z: V \rightarrow \mathbb{R}$, it is understood that $\mathrm{B}_{\ell}(i ; G)$ inherits the 'same' marks. We will write in this case $\left(\mathrm{B}_{\ell}(i ; G), z\right)$ instead of the cumbersome (but more explicit) notation $\left(\mathrm{B}_{\ell}(i ; G), i, z_{\mathrm{B}_{\ell}(i ; G)}\right)$.

Definition 2.1. A radius- $\ell$ local algorithm for the semidefinite program (3) is any measurable function $F: \mathcal{G}^{*} \rightarrow \mathbb{R}$ such that

1. $F\left(G_{1},{ }_{1}, z_{1}\right)=F\left(G_{2}, 2, z_{2}\right)$ if $\left(\mathrm{B}_{\ell}\left({ }_{1} ; G_{1}\right), z_{1}\right)$ is isomorphic to $\left(\mathrm{B}_{\ell}\left({ }_{2} ; G_{2}\right), z_{2}\right)$ under graph isomorphism that preserves the root vertex and vertex marks.

2. If $z=(z(i))_{i \in V}$ are i.i.d. and distributed as $z(i) \sim \operatorname{Normal}(0,1)$, then $\mathbb{E}_{\boldsymbol{z}}\left\{F(G,, z)^{2}\right\}=1$. (Here and below $\mathbb{E}_{\boldsymbol{z}}$ denotes expectation with respect to the random variables $z$ ).

We denote the set of such functions $F$ by $\mathcal{F}_{*}(\ell)$.

A local algorithm is a radius- $\ell$ local algorithm for some fixed $\ell$ (independent of the graph). The set of such functions is denoted by $\mathcal{F}_{*} \equiv \cup_{\ell \geq 1} \mathcal{F}_{*}(\ell)$.

We apply a local algorithm to a fixed graph $G$ by generating i.i.d. marks $z=(z(i))_{i \in V}$ as $z(i) \sim \operatorname{Normal}(0,1)$, and producing the random variable $\xi_{i}=F(G, i, z)$ for each vertex $i \in V$. In other words, we use the radius- $\ell$ local algorithm to compute, for each vertex of $G$, a function of the ball of radius $\ell$ around that vertex that depends on the additional randomness provided by the $z(i)$ 's in this ball. The covariance matrix $X=\mathbb{E}_{\boldsymbol{z}}\left\{\xi \xi^{\top}\right\}$ is a feasible point 
for the SDP (3), achieving the value $n \mathcal{E}(F ; G)$ where

$$
\mathcal{E}(F ; G) \equiv \frac{1}{n} \mathbb{E}_{\boldsymbol{z}}\left\{\sum_{i, j \in V}\left(\boldsymbol{A}_{i j}-\frac{d}{n}\right) F(G, i, \boldsymbol{z}) F(G, j, \boldsymbol{z})\right\} .
$$

It is a well-known elementary fact that any feasible point for the SDP (3) can be written as $X=\mathbb{E}\left\{\xi \xi^{\top}\right\}$, for $\xi$ a random vector in $\mathbb{R}^{n}$ with $\mathbb{E}\left\{\xi_{i}^{2}\right\}=1$. However, it is surprising that the SDP value can be approximated by restricting ourselves to random vectors $\xi$ that are produced by local algorithms, when the underlying graph $G$ is random.

We are now in position to state our main results.

\subsection{Erdős-Rényi Random Graphs $G \sim \mathrm{G}(n, d / n)$}

We first prove an optimality guarantee, in the large $n$ limit, for the value achieved by a simple local algorithm (or more precisely, a sequence of simple local algorithms) solving (3) on the Erdős-Rényi graph.

Theorem 2.2. Fix $d \geq 0$ and let $A=A_{G_{n}}$ be the adjacency matrix of the Erdös-Rényi random graph $G_{n} \sim \mathrm{G}(n, d / n)$. Then for $d<1$, almost surely,

$$
\lim _{n \rightarrow \infty} \frac{1}{n} \operatorname{SDP}(A)=d
$$

For $d \geq 1$, almost surely,

$$
\begin{aligned}
2 \sqrt{d} & \left(1-\frac{1}{d+1}\right) \leq \liminf _{n \rightarrow \infty} \frac{1}{n} \operatorname{SDP}(A) \\
& \leq \limsup _{n \rightarrow \infty} \frac{1}{n} \operatorname{SDP}(A) \leq 2 \sqrt{d}\left(1-\frac{1}{2 d}\right) .
\end{aligned}
$$

Furthermore, there exists a sequence of local algorithms that achieves the lower bound. Namely, for each $\varepsilon>0$, there exist $\ell(\varepsilon)>0$ and $F \in \mathcal{F}_{*}(\ell(\varepsilon))$ such that, almost surely,

$$
\lim _{n \rightarrow \infty} \mathcal{E}\left(F ; G_{n}\right) \geq 2 \sqrt{d}\left(1-\frac{1}{d+1}\right)-\varepsilon .
$$

As anticipated in the introduction, the upper and lower bounds of (5) approach each other for large $d$, implying in particular $\operatorname{SDP}(A) / n=$ $2 \sqrt{d}(1-\Theta(1 / d))+o_{n}(1)$. This should be compared with the result of [MS16] yielding $\operatorname{SDP}(A) / n=2 \sqrt{d}\left(1+o_{d}(1)\right)+o_{n}(1)$. Also, by simple calculus, the upper and lower bounds stay within a ratio bounded by $8 / 9$ for all $d$, with the worst-case ratio $8 / 9$ being achieved at $d=2$. Finally, they again converge as $d \rightarrow 1$, implying in particular $\lim _{n \rightarrow \infty} \operatorname{SDP}(A) / n=1$ for $d=1$.

REMARK 2.3. The result $\lim _{n \rightarrow \infty} \operatorname{SDP}(A) / n=d$ for $d<1$ is elementary and only stated for completeness. Indeed, for $<1$, the graph $G \sim \mathrm{G}(n, d / n)$ decomposes with high probability into disconnected components of size $O(\log n)$, which are all trees or unicyclic [fLR11]. As a consequence, the vertex set can be partitioned into two subsets of size $n / 2$ so that at most one connected component of $G$ has vertices on both sides of the partition, and hence at most two edges cross the partition. By using the feasible point $X=\sigma \sigma^{\top}$ with $\sigma \in\{+1,-1\}^{n}$ the indicator vector of the partition, we get $2|E|-8 \leq \operatorname{SDP}(A) \leq 2|E|$ whence the claim follows immediately.

The local algorithm achieving the lower bound of Theorem 2.2 is extremely simple. At each vertex $i \in V$, it outputs a weighted sum of the random variables $z(j)$ with $j \in \mathrm{B}_{\ell}(i ; G)$, with weight proportional to $d^{-\operatorname{dist}(i, j) / 2}$ (here $\operatorname{dist}(i, j)$ is the graph distance between vertices $i$ and $j$ ). This can be regarded as a special case of a general scheme that sums over the random variables $z(j), j \in$ $\mathrm{B}_{\ell}(i ; G)$, with weight $\operatorname{br}(T)^{-\operatorname{dist}(i, j) / 2}$, where $\operatorname{br}(T)$ is the branching number of the tree that corresponds to the local weak limit of the graphs $G$. When applied to random $d$-regular graphs (the resulting weights being $\left.(d-1)^{-\operatorname{dist}(i, j) / 2}\right)$, this approach is related to the Gaussian wave function of [CGHV15] and is known to achieve the value $\operatorname{SDP}(\boldsymbol{A})$ in the large $n$ limit [MS16].

\subsection{A Local Algorithm Based on Harmonic Measures}

A natural question arising from the previous section is whether a better local algorithm can be constructed by summing the random variables $z(j)$ with different weights, to account for the graph geometry. It turns out that indeed this is possible by using a certain harmonic weighting scheme that we next describe, deferring some technical details to Appendix C. This scheme is inspired by the non-rigorous calculations of [JMRT16] that uses the 'cavity method' from statistical physics: it would be interesting to explore the connection in other models as well. Throughout we assume $d>1$.

Recall that the random graph $G \sim \mathrm{G}(n, d / n)$ converges locally to a Galton-Watson tree (see Appendix $\mathrm{C}$ for background on local weak convergence). This can be shown to imply that it is sufficient to define the function $F \in \mathcal{F}_{*}$ for trees. Let $(T$,$) be an infinite$ rooted tree and consider the simple random walk on $T$ started at, which we assume to be transient. The harmonic measure assigns to vertex $v \in V(T)$, with $\operatorname{dist}(, v)=k$, a weight $h^{()}(v)$ which is the probability ${ }^{3}$ that the walk exits for the last time $\mathrm{B}_{k}(; T)$ at $v$ [LPP95]. We then define

$$
\widetilde{F}(T,, z) \equiv \frac{1}{\sqrt{\ell+1}} \sum_{v \in \mathrm{B}_{\ell}(, T)} \sqrt{h^{()}(v)} z(v) .
$$

Technically speaking, this is not a local function because the weights $h^{()}(v)$ depend on the whole tree T. However a local approximation to these weights can be constructed by truncating $T$ at a depth $L \geq \ell$ : details are provided in Appendix C.

Given the well-understood relationship between random walks and electrical networks, it is not surprising that the value achieved by this local algorithm can be expressed in terms of conductance. The conductance $\mathrm{c}(T$,$) of a rooted tree (T$,$) is the intensity of$ current flowing out of the root when a unit potential difference is imposed between the root and the boundary ('at infinity'). It is understood that $\mathrm{c}(T)=$,0 if $T$ is finite.

THEOREM 2.4. For $(T$,$) a Galton-Watson tree with offspring distri-$ bution Poisson $(d)$, let $\mathrm{c}_{1}, \mathrm{c}_{2} \stackrel{\mathrm{d}}{=} \mathrm{c}(T$, ) be two independent and identically distributed copies of the conductance of T. Let $A=A_{G_{n}}$ be the adjacency matrix of the Erdös-Rényi random graph $G_{n} \sim \mathrm{G}(n, d / n)$.

\footnotetext{
${ }^{3}$ For each distance $k$, the weights $h^{()}(v)$ form a probability distribution over vertices at distance $k$ from the root. These distributions can be derived from a unique probability measure over the boundary of $T$ at infinity, as is done in [LPP95], but this is not necessary here.
} 
Then for $d>1$, almost surely,

$$
\begin{gathered}
\liminf _{n \rightarrow \infty} \frac{1}{n} \operatorname{SDP}(A) \geq d \mathbb{E} \Psi\left(\mathrm{c}_{1}, \mathrm{c}_{2}\right), \\
\Psi\left(\mathrm{c}_{1}, \mathrm{c}_{2}\right) \equiv \begin{cases}\frac{\mathrm{c}_{1} \sqrt{1+\mathrm{c}_{2}}+\mathrm{c}_{2} \sqrt{1+\mathrm{c}_{1}}}{\mathrm{c}_{1}+\mathrm{c}_{2}+\mathrm{c}_{1} \mathrm{c}_{2}} & \text { if } \mathrm{c}_{1}>0 \text { or } \mathrm{c}_{2}>0, \\
1 & \text { otherwise. }\end{cases}
\end{gathered}
$$

Furthermore, for each $\varepsilon>0$, there exist $\ell(\varepsilon)>0$ and $F \in \mathcal{F}_{*}(\ell(\varepsilon))$ such that, almost surely,

$$
\lim _{n \rightarrow \infty} \mathcal{E}\left(F ; G_{n}\right) \geq d \mathbb{E} \Psi\left(\mathrm{c}_{1}, \mathrm{c}_{2}\right)-\varepsilon .
$$

Finally, for large d, this lower bound behaves as

$$
d \mathbb{E} \Psi\left(\mathrm{c}_{1}, \mathrm{c}_{2}\right)=2 \sqrt{d}\left(1-\frac{5}{8 d}+O\left(\left(\frac{\log d}{d}\right)^{3 / 2}\right)\right) .
$$

The lower bound $d \mathbb{E} \Psi\left(\mathrm{c}_{1}, \mathrm{c}_{2}\right)$ is not explicit but can be efficiently evaluated numerically, by sampling the distributional recursion satisfied by c. This numerical technique was used in [JMRT16], to which we refer for further details. The result of such a numerical evaluation is plotted as the lower solid line in Figure 1. This harmonic lower bound seems to capture extremely well our numerical data for $\operatorname{SDP}(A)$ (red circles).

Note that Theorem 2.4 implies that the lower bound in Theorem 2.2 is not tight (see in particular (11)) and it provides a tighter lower bound (at least for large $d$ ).

\subsection{Stochastic Block Model $G \sim \mathrm{G}(n, a / n, b / n)$}

As discussed in the previous sections, local algorithms can approximately solve the SDP (3) for $\boldsymbol{A}=\boldsymbol{A}_{G}$ the adjacency matrix of $G \sim \mathrm{G}(n, d / n)$. The stochastic block model $G \sim \mathrm{G}(n, a / n, b / n)$ provides a simple example in which they are bound to fail, although they can succeed with a small amount of additional side information.

As stated in the introduction, $G \sim \mathrm{G}(n, a / n, b / n)$ is a random graph over $n$ vertices generated as follows. Let $\sigma \in\{+1,-1\}^{n}$ be distributed uniformly at random, conditional on $\langle\sigma, 1\rangle=0$. Conditional on $\sigma$, any two vertices $i, j$ are connected by an edge independently with probability $a / n$ if $\sigma(i)=\sigma(j)$ and with probability $b / n$ otherwise. We will assume $a>b$ : in the social sciences parlance, the graph is assortative.

The average vertex degree of such a graph is $d=(a+b) / 2$. We assume $d>1$ to ensure that $G$ has a giant component with high probability. The signal-to-noise ratio parameter $\lambda=(a-$ $b) / \sqrt{2(a+b)}$ plays a special role in the model's behavior. If $\lambda<1$, then the total variation distance between $\mathrm{G}(n, a / n, b / n)$ and the Erdős-Rényi graph $\mathrm{G}(n, d / n)$ is bounded away from 1 . On the other hand, if $\lambda \geq 1$, then we can test whether $G \sim \mathrm{G}(n, a / n, b / n)$ or $G \sim \mathrm{G}(n, d / n)$ with probability of error converging to 0 as $n \rightarrow \infty$ [MNS12].

The next theorem lower-bounds the SDP value for the stochastic block model.

Theorem 2.5. Let $\boldsymbol{A}=\boldsymbol{A}_{G_{n}}$ be the adjacency matrix of the random graph $G_{n} \sim \mathrm{G}(n, a / n, b / n)$. If $d=(a+b) / 2>1$ and $\lambda=(a-$ $b) / \sqrt{2(a+b)}>1$, then for a universal constant $C>0$ (independent of $a$ and $b$ ), almost surely,

$$
\liminf _{n \rightarrow \infty} \frac{1}{n} \operatorname{SDP}(A) \geq \sqrt{d} \max \left(\lambda, 2+\frac{(\lambda-1)^{2}}{\lambda \sqrt{d}}-\frac{C}{d}\right) .
$$

(The first bound in (12) dominates for large $\lambda$, whereas the second dominates near the information-theoretic threshold $\lambda=1$ for large d.)

On one hand, this theorem implies that local algorithms fail to approximately solve the SDP (3) for the stochastic block model, for the following reason: The local structures of $G \sim \mathrm{G}(n, a / n, b / n)$ and $G \sim \mathrm{G}(n, d / n)$ are the same asymptotically, in the sense that they both converge locally to the Galton-Watson tree with Poisson $(d)$ offspring distribution. This and the upper bound of Theorem 2.2 immediately imply that for any $F \in \mathcal{F}_{*}$,

$$
\limsup _{n \rightarrow \infty} \mathcal{E}\left(F ; G_{n}\right) \leq 2 \sqrt{d}\left(1-\frac{1}{2 d}\right) .
$$

In particular, the gap between this upper bound and the lower bound (12) for the SDP value is unbounded for large $\lambda$.

This problem is related to the symmetry between +1 and -1 labels in this model. It can be resolved if we allow the local algorithm to depend on $\mathrm{B}_{\ell_{n}}(; G)$ where $\ell_{n}$ grows logarithmically in $n$, or alternatively if we provide a small amount of side information about the hidden partition. Here we explore the latter scenario (see also [ZMZ14, MX16] for related work).

Suppose that for each vertex $i \in V$, the label $\sigma(i) \in\{+1,-1\}$ is revealed independently with probability $\delta$ for some fixed $\delta>0$, and that the radius $-\ell$ local algorithm has access to the revealed labels in $\mathrm{B}_{\ell}(; G)$. More formally, let $\mathcal{M}=\{+1,-1, \mathrm{U}\}$ be the set of possible vertex labels, where $U$ codes for 'unrevealed', let $\sigma: V \rightarrow \mathcal{M}$ be any assignment of labels to vertices, and let $\mathcal{G}^{*}(\mathcal{M})$ be the space of tuples $(G,, \sigma, z)$ (where $(G,, z) \in \mathcal{G}^{*}$ as before).

Definition 2.6. A radius- $\ell$ local algorithm using partially revealed labels for the semidefinite program (3) is any measurable function $F: \mathcal{G}^{*}(\mathcal{M}) \rightarrow \mathbb{R}$ such that

$$
\text { 1. } \begin{aligned}
F\left(G_{1},{ }_{1}, z_{1}, \sigma_{1}\right)=F\left(G_{2}, 2, z_{2}, \sigma_{2}\right) \text { if } \\
\left(\mathrm{B}_{\ell}\left({ }_{1} ; G_{1}\right), z_{1}, \sigma_{1}\right) \simeq\left(\mathrm{B}_{\ell}\left({ }_{2} ; G_{2}\right), z_{2}, \sigma_{2}\right),
\end{aligned}
$$

where $\simeq$ denotes isomorphism that preserves the root vertex, vertex marks, and vertex labels in $\mathcal{M}$.

2. If $z=(z(i))_{i \in V}$ are i.i.d. and distributed as $z(i) \sim \operatorname{Normal}(0,1)$, then $\mathbb{E}_{\boldsymbol{z}}\left\{F(G,, \boldsymbol{\sigma}, \boldsymbol{z})^{2}\right\}=1$, where $\mathbb{E}_{\boldsymbol{z}}$ denotes expectation only over $z$.

We denote the set of such functions $F$ by $\mathcal{F}_{*}{ }^{\mathcal{M}}(\ell)$, and we denote $\mathcal{F}_{*}^{\mathcal{M}} \equiv \cup_{\ell \geq 1} \mathcal{F}_{*}{ }^{\mathcal{M}}(\ell)$. For any $F \in \mathcal{F}_{*}{ }^{\mathcal{M}}$, we denote

$$
\begin{aligned}
& \mathcal{E}(F ; G, \boldsymbol{\sigma}) \equiv \\
& \frac{1}{n} \mathbb{E}_{\boldsymbol{z}}\left\{\sum_{i, j \in V}\left(\boldsymbol{A}_{i j}-\frac{d}{n}\right) F(G, i, \boldsymbol{\sigma}, \boldsymbol{z}) F(G, j, \boldsymbol{\sigma}, \boldsymbol{z})\right\},
\end{aligned}
$$

so that $F$ yields a solution to the $\operatorname{SDP}(3)$ achieving value $n \mathcal{E}(F ; G ; \sigma)$. Then we have the following result:

Theorem 2.7. Let $A=A_{G_{n}}$ be the adjacency matrix of the random graph $G_{n} \sim \mathrm{G}(n, a / n, b / n)$. For any fixed $\delta>0$, let $\sigma_{n}=$ $\left(\sigma_{n}(i)\right)_{i \in V\left(G_{n}\right)}$ be random and such that, independently for each $i \in V\left(G_{n}\right)$, with probability $1-\delta$ we have $\sigma_{n}(i)=\mathrm{U}$ (the label is 
unrevealed), and with probability $\delta$ we have that $\sigma_{n}(i) \in\{+1,-1\}$ identifies the component of the hidden partition containing $i$. If $d=$ $(a+b) / 2 \geq 2$ and $\lambda=(a-b) / \sqrt{2(a+b)}>1$, then for any $\varepsilon>0$, there exist $\ell(\varepsilon)>0$ and $F \in \mathcal{F}_{*}^{\mathcal{M}}(\ell(\varepsilon))$ for which, almost surely,

$$
\lim _{n \rightarrow \infty} \mathcal{E}\left(F ; G_{n}, \sigma_{n}\right) \geq \sqrt{d}\left(2+\frac{(\lambda-1)^{2}}{\lambda \sqrt{d}}-\frac{C}{d}\right)-\varepsilon .
$$

The restriction to $d \geq 2$ above is arbitrary; our proof is valid if this constraint is relaxed to $d \geq 1+\eta$ for any $\eta>0$, at the expense of a larger constant $C:=C_{\eta}$. This theorem implies the second lower bound of (12) when $d \geq 2$; the first lower bound of (12) is trivial and proven in Appendix C.

\subsection{Testing in the Stochastic Block Model}

Semidefinite programming can be used as follows to test whether $G \sim \mathrm{G}(n, a / n, b / n)$ or $G \sim \mathrm{G}(n, d / n):$

1. Given the graph $G$, compute the value $\operatorname{SDP}\left(A_{G}\right)$ of the program (3).

2. If $\operatorname{SDP}\left(A_{G}\right) / n \geq 2 \sqrt{d}\left(1-(2 d)^{-1}\right)+\varepsilon$, reject the null hypothesis $G \sim \mathrm{G}(n, d / n)$.

(Here $\varepsilon$ is a small constant independent of $n$.) The rationale for this procedure is provided by Theorem 2.2, implying that, if $G \sim$ $\mathrm{G}(n, d / n)$, then the probability of false discovery (i.e. rejecting the null when $G \sim \mathrm{G}(n, d / n))$ converges to 0 as $n \rightarrow \infty$.

We have the following immediate consequence of Theorem 2.2 and Theorem 2.5 (here error probability refers to the probability of false discovery plus the probability of miss-detection, i.e. not rejecting the null when $G \sim \mathrm{G}(n, a / n, b / n))$ :

COROLLARY 2.8. The SDP-based test has error probability converging to 0 provided $\lambda>\lambda^{\mathrm{SDP}}(d)$, where

$$
\lambda^{\mathrm{SDP}}(d) \leq \min \left(2-\frac{1}{d}, 1+\frac{C}{d^{1 / 4}}\right) .
$$

For comparison ${ }^{4}$, [MS16] proved $\lambda^{\mathrm{SDP}}(d)=1+o_{d}(1)$ for large $d$, while the last result gives a quantitative bound for all $d$.

\section{FURTHER RELATED WORK}

The SDP relaxation (3) has attracted a significant amount of work since Goemans-Williamson's seminal work on the MAXCUT problem [GW95]. In the last few years, several authors used this approach for clustering or community detection and derived optimality or near-optimality guarantees. An incomplete list includes [BCSZ14, ABH16, HWX16, HWX15, ABC $\left.{ }^{+} 15\right]$. Under the assumption that $G$ is generated according to the stochastic block model (whose two-groups version was introduced in Section 2.3), these papers provide conditions under which the SDP approach recovers exactly the vertex labels. This can be regarded as a 'high signal-tonoise ratio' regime, in which (with high probability) the SDP solution has rank one and is deterministic (i.e. independent of the graph realization). In contrast, we focus on the 'pure noise' scenario in which $G \sim \mathrm{G}(n, d / n)$ is an Erdős-Rényi random graph, or on the twogroups stochastic block-model $G \sim \mathrm{G}(n, a / n, b / n)$ close to the detection threshold. In this regime, the SDP optimum has rank larger

\footnotetext{
${ }^{4}$ Note [MS16] also proves guarantees on the estimation error. We believe it should be possible to improve those results using the methods in the present paper, but we defer it to future work.
}

than one and is non-deterministic. Previous papers that have studied this regime using SDP include [GV15, JMRT16, MS16, MPW16]. For $G \sim \mathrm{G}(n, d / n)$, [DMS15] established the asymptotic value of the minimum bisection problem (2) in the large- $d$ limit, and the results of [MS16] (as well as Theorem 2.2 of this paper) imply that the optimal value of the SDP relaxation is larger by a factor of approximately 1.310 for large $d$.

Several papers applied sophisticated spectral methods to the stochastic block model near the detection threshold $\left[\mathrm{KMM}^{+} 13\right.$, Mas14, MNS13, BLM15]. Our upper bound in Theorem 2.2 establishes feasibility of a certain dual witness construction using an argument similar to [BLM15]. It has been noted that the SDP approach may be more robust to adversarial perturbations of the graph [JMRT16, MS16], and this was studied in greater detail in the context of a semirandom model with monotone adversary in [MPW16].

Several papers studied the use of local algorithms to solve combinatorial optimization problems on graphs. Hatami, Lovász and Szegedy [HLS14] investigated several notions of graph convergence, and put forward a conjecture implying, in particular, that local algorithms are able to find (nearly) optimal solutions of a broad class of combinatorial problems on random $d$-regular graphs. This conjecture was disproved by Gamarnik and Sudan [GS14] by considering maximum size independent sets on random $d$-regular graphs. In particular, they proved that the size of an independent set produced by a local algorithm is at most $(1 / 2)+(1 / \sqrt{8})+\varepsilon$ times the maximum independent set, for large enough $d$. Rahman and Virag [RV14] improved this result by establishing that no local algorithm can produce independent sets of size larger than $(1 / 2)+\varepsilon$ times the maximum independent set, for large enough $d$. This approximation ratio is essentially optimal, since known local algorithms can achieve $(1 / 2)-\varepsilon$ of the maximum independent set. It is unknown whether a similar gap is present for small degree $d$. In particular, Csóka et al. [CGHV15] establish a lower-bound on the max-size independent set on random 3-regular graphs. A similar technique is used by Lyons [Lyo14] to lower bound the max-cut on random 3-regular graphs. In summary, the question of which graph-structured optimization problems can be approximated by local algorithms is broadly open.

By construction, local algorithms can be applied to infinite random graphs, and have a well defined value provided the graph distribution is unimodular (see below). Asymptotic results for graph sequences can be 'read-off' these infinite-graph settings (our proofs will use this device multiple times). In this context, the (random) solutions generated by local algorithms, together with their limits in the weak topology, are referred to as 'factors of i.i.d. processes' [Lyo14].

\section{A NOTATION}

We provide proof outlines in these appendices; complete proofs may be found in the full version of this paper.

We use upper case boldface for matrices (e.g. $A, B$ ), lower case boldface for vectors (e.g. $\boldsymbol{u}, \boldsymbol{v})$ and lower case plain for scalars (e.g. $x, y$ ). The scalar product of vectors $\boldsymbol{u}, \boldsymbol{v} \in \mathbb{R}^{m}$ is denoted by $\langle\boldsymbol{u}, \boldsymbol{v}\rangle=\sum_{i=1}^{m} u_{i} v_{i}$, and the scalar product between matrices is indicated in the same way $\langle\boldsymbol{A}, \boldsymbol{B}\rangle=\operatorname{Tr}\left(\boldsymbol{A} \boldsymbol{B}^{\mathrm{T}}\right)$. 
Given a matrix $A \in \mathbb{R}^{m \times m}, \operatorname{diag}(A) \in \mathbb{R}^{m}$ is the vector that contains its diagonal entries. Conversely, given $\boldsymbol{v} \in \mathbb{R}^{m}, \operatorname{diag}(\boldsymbol{v}) \in$ $\mathbb{R}^{m \times m}$ is the diagonal matrix with entries $\operatorname{diag}(\boldsymbol{v})_{i i}=v_{i}$.

We denote by 1 the all-ones vector and by Id the identity matrix.

We follow the standard big-Oh notation.

\section{B UPPER BOUND}

We outline the proof of the upper bound in Theorem 2.2. Denote $\mathrm{PSD}_{1}:=\left\{X: X \geq 0, X_{i i}=1 \forall i\right\}$. Introducing dual variables $\Lambda \geq 0$ and $v \in \mathbb{R}^{n}$ and invoking strong duality, we have

$$
\begin{aligned}
& \operatorname{SDP}(A) \\
& \begin{aligned}
=\max _{\boldsymbol{X} \in \mathrm{PSD}_{1}} \min _{\boldsymbol{v}, \boldsymbol{\Lambda}}\left\langle\boldsymbol{A}-\frac{d}{n} \mathbf{1 1}^{\top}, \boldsymbol{X}\right\rangle & +\langle\boldsymbol{\Lambda}, \boldsymbol{X}\rangle \\
& -\langle\boldsymbol{v}, \operatorname{diag}(\boldsymbol{X})-\mathbf{1}\rangle
\end{aligned} \\
& =\min _{\boldsymbol{v}, \boldsymbol{\Lambda}} \max _{\boldsymbol{X} \in \mathrm{PSD}_{1}}\langle\boldsymbol{v}, \mathbf{1}\rangle+\left\langle\boldsymbol{A}-\frac{d}{n} \mathbf{1 1}^{\top}+\boldsymbol{\Lambda}-\operatorname{diag}(\boldsymbol{v}), \boldsymbol{X}\right\rangle .
\end{aligned}
$$

The minimum over $\Lambda \geq 0$ occurs at $\Lambda=0$, hence $\operatorname{SDP}(A)$ is equivalently given by the value of the dual minimization problem over $v \in \mathbb{R}^{n}:$

$$
\begin{aligned}
\text { minimize } & \langle\boldsymbol{v}, \mathbf{1}\rangle \\
\text { subject to } & A-\frac{d}{n} \mathbf{1 1}^{\top} \leq \operatorname{diag}(\boldsymbol{v}) .
\end{aligned}
$$

We prove the upper bound in Theorem 2.2 by constructing a dual-feasible solution $\boldsymbol{v}$, parametrized by a small positive constant $\delta \in(0,1 / \sqrt{d})$. Denote the diagonal degree matrix of $\boldsymbol{A}$ as $\boldsymbol{D}:=$ $\operatorname{diag}(\boldsymbol{A 1})$ and set

$$
\begin{aligned}
u & :=\frac{1}{\sqrt{d}}-\delta \\
\boldsymbol{H} & :=\left(1-u^{2}\right) \operatorname{Id}+u^{2} \boldsymbol{D}-u \boldsymbol{A} \\
\boldsymbol{v} & := \begin{cases}\operatorname{diag}\left(\frac{1+\delta-u^{2}}{u} \mathrm{Id}+u \boldsymbol{D}\right) & \text { if } \frac{\boldsymbol{H}+\delta \mathrm{Id}}{u}+\frac{d}{n} \mathbf{1 1}^{\top} \geq 0 \\
\operatorname{diag}(\boldsymbol{D}) & \text { otherwise. }\end{cases}
\end{aligned}
$$

The upper bound is a consequence of the following result, which ensures that the first case in the definition of $v$ in (18) holds with high probability.

Theorem B.1. For fixed $d>1$, let $\boldsymbol{A}$ be the adjacency matrix of the Erdös-Rényi random graph $G \sim \mathrm{G}(n, d / n)$, and let $\boldsymbol{D}:=\operatorname{diag}(\boldsymbol{A 1})$ be the diagonal degree matrix. Then for any $\delta \in(0,1 / \sqrt{d})$ and for $u=1 / \sqrt{d}-\delta$, with probability approaching 1 as $n \rightarrow \infty$,

$$
\frac{1+\delta-u^{2}}{u} \mathrm{Id}+u D-A+\frac{\left(1-u^{2}\right) d}{n} 1^{\top}>0 .
$$

Let us first show that this implies the desired bound:

Proof SKetch of Theorem 2.2 (Upper bound). $v$ as defined in (18) is a feasible solution for the dual problem (17). Let $\mathcal{E}$ be the event where

$$
\frac{1+\delta-u^{2}}{u} \operatorname{Id}+u D-A+\frac{d}{n} \mathbf{1 1}^{\top} \geq 0 .
$$

Then

$$
\boldsymbol{v}^{\top} \mathbf{1}=\left(\frac{1+\delta-u^{2}}{u} n+u \mathbf{1}^{\top} \boldsymbol{A} \mathbf{1}\right) \mathbb{1}\{\mathcal{E}\}+\left(\mathbf{1}^{\top} \boldsymbol{A} \mathbf{1}\right) \mathbb{1}\left\{\mathcal{E}^{c}\right\} .
$$

As $\mathbf{1}^{\top} A \mathbf{1} \sim 2 \operatorname{Binom}\left(\left(\begin{array}{l}n \\ 2\end{array}\right), d / n\right)$, this implies $\mathbb{E}\left[\mathbf{1}^{\top} A 1\right] \leq d n$ and $\mathbb{E}\left[\left(\mathbf{1}^{\top} \boldsymbol{A} \mathbf{1}\right)^{2}\right] \leq d^{2}\left(n^{2}+1\right)$. Then

$$
\begin{aligned}
& \mathbb{E}\left[\frac{1}{n} \operatorname{SDP}(A)\right] \leq \frac{1}{n} \mathbb{E}\left[\boldsymbol{v}^{\top} \mathbf{1}\right] \\
& \leq \frac{1+\delta-u^{2}}{u}+\frac{u}{n} \mathbb{E}\left[\mathbf{1}^{\top} \boldsymbol{A 1}\right]+\frac{1}{n} \mathbb{E}\left[\left(\mathbf{1}^{\top} \boldsymbol{A} \mathbf{1}\right) \mathbb{1}\left\{\mathcal{E}^{c}\right\}\right] \\
& \leq \frac{1+\delta-u^{2}}{u}+u d+\frac{1}{n} \sqrt{\mathbb{E}\left[\left(\mathbf{1}^{\top} \boldsymbol{A} \mathbf{1}\right)^{2}\right] \mathbb{P}\left[\mathcal{E}^{c}\right]} \\
& \leq \frac{1+\delta-u^{2}}{u}+u d+d \sqrt{\mathbb{P}\left[\mathcal{E}^{c}\right]} .
\end{aligned}
$$

By Theorem B.1, $\mathbb{P}\left[\mathcal{E}^{c}\right] \rightarrow 0$ as $n \rightarrow \infty$. Taking $n \rightarrow \infty$ and then $\delta \rightarrow 0$,

$$
\limsup _{n \rightarrow \infty} \mathbb{E}\left[\frac{1}{n} \operatorname{SDP}(A)\right] \leq 2 \sqrt{d}-\frac{1}{\sqrt{d}}=2 \sqrt{d}\left(1-\frac{1}{2 d}\right) .
$$

The proof may be completed by establishing concentration of $\frac{1}{n} \operatorname{SDP}(A)$ around its mean.

Turning to Theorem B.1, heuristically we expect this result to be true by the following reasoning: The matrix $\boldsymbol{H}=\boldsymbol{H}(u)$ is the deformed Laplacian, or Bethe Hessian, of the graph. By a relation in graph theory known as the Ihara-Bass formula [Bas92, KS00], the values of $u$ for which this matrix is singular are the inverses of the non-trivial eigenvalues of a certain "non-backtracking matrix" $\left[\mathrm{KMM}^{+} 13, \mathrm{SKZ14}\right]$. Theorem 3 of [BLM15] shows that this non-backtracking matrix has, with high probability, the bulk of its spectrum supported on the complex disk of radius approximately $\sqrt{d}$, with a single outlier eigenvalue at $d$. From this, the observation that $\boldsymbol{H}(0) \geq 0$, and a continuity argument in $u$, one deduces that $\boldsymbol{H}(u)$ has, with high probability for large $n$, only a single negative eigenvalue when $u \in(1 / d, 1 / \sqrt{d})$. If the eigenvector corresponding to this eigenvalue has positive alignment with $1 \in \mathbb{R}^{n}$, then adding a certain multiple of the rank-one matrix $11^{\top}$ should eliminate this negative eigenvalue.

Direct analysis of the rank-one perturbation of $\boldsymbol{H}(u)$ is hindered by the fact that the spectrum and eigenvectors of $\boldsymbol{H}(u)$ are difficult to characterize. Instead, we study a certain perturbation of the nonbacktracking matrix. We prove Theorem B.1 via the following two steps: First, we prove a generalization of the Ihara-Bass relation to edge-weighted graphs. For any graph $H=(V, E)$, let $c: E \rightarrow \mathbb{R}$ be a set of possibly negative edge weights. For each $u \in \mathbb{R}$ such that $|u| \notin\left\{|c(i, j)|^{-1}:\{i, j\} \in E\right\}$, define the $n \times n$ symmetric matrix $\boldsymbol{A}_{c, u}$ and diagonal matrix $D_{c, u}$ by

$$
\begin{aligned}
& \boldsymbol{A}_{c, u}(i, j)= \begin{cases}\frac{u c(i, j)}{1-u^{2} c(i, j)^{2}} & \{i, j\} \in E \\
0 & \text { otherwise, }\end{cases} \\
& \boldsymbol{D}_{c, u}(i, j)= \begin{cases}\sum_{k:\{i, k\} \in E} \frac{u^{2} c(i, k)^{2}}{1-u^{2} c(i, k)^{2}} & i=j \\
0 & \text { otherwise. }\end{cases}
\end{aligned}
$$

Let $E^{o}$ denote the set of directed edges $E^{o}:=\{(i, j),(j, i):\{i, j\} \in E\}$, and define the weighted non-backtracking matrix $\boldsymbol{B}_{c}$, with rows and columns indexed by $E^{o}$, as

$$
\boldsymbol{B}_{c}\left((i, j),\left(i^{\prime}, j^{\prime}\right)\right)= \begin{cases}c\left(i^{\prime}, j^{\prime}\right) & i^{\prime}=j, j^{\prime} \neq i \\ 0 & \text { otherwise }\end{cases}
$$


The following result relates $\boldsymbol{B}_{c}$ with a generalized deformed Laplacian defined by $\boldsymbol{A}_{c, u}$ and $\boldsymbol{D}_{c, u}$ :

Lemma B.2 (Generalized Ihara-Bass Formula). For any graph $H=(V, E)$, edge weights $c: E \rightarrow \mathbb{R}, u \in \mathbb{R}$ with $|u| \notin\left\{|c(i, j)|^{-1}:\right.$ $\{i, j\} \in E\}$, and the matrices $\boldsymbol{B}_{c}, \boldsymbol{A}_{c, u}$, and $\boldsymbol{D}_{c, u}$ as defined above,

$$
\begin{aligned}
& \operatorname{det}\left(\operatorname{Id}-u \boldsymbol{B}_{c}\right) \\
& =\operatorname{det}\left(\operatorname{Id}+\boldsymbol{D}_{c, u}-\boldsymbol{A}_{c, u}\right) \prod_{\{i, j\} \in E}\left(1-u^{2} c^{2}(i, j)\right) .
\end{aligned}
$$

When $c \equiv 1$, this recovers the standard Ihara-Bass identity.

Second, we consider a weighted non-backtracking matrix $\boldsymbol{B} \in$ $\mathbb{R}^{n(n-1) \times n(n-1)}$ of the above form for the complete graph with $n$ vertices, with rows and columns indexed by all ordered pairs $(i, j)$ of distinct indices $i, j \in\{1, \ldots, n\}$, and defined as

$$
\boldsymbol{B}\left((i, j),\left(i^{\prime}, j^{\prime}\right)\right)= \begin{cases}A_{i^{\prime} j^{\prime}}-\frac{d}{n} & i^{\prime}=j, j^{\prime} \neq i \\ 0 & \text { otherwise. }\end{cases}
$$

We show that $\boldsymbol{B}$ no longer has an outlier eigenvalue at $d$, but instead has all of its eigenvalues contained within the complex disk of radius approximately $\sqrt{d}$ :

Lemma B.3. Fix $d>1$, let $A$ be the adjacency matrix of the ErdösRényi random graph $\mathrm{G}(n, d / n)$, and define $\boldsymbol{B} \in \mathbb{R}^{n(n-1) \times n(n-1)}$ by (20). Let $\rho(\boldsymbol{B})$ denote the spectral radius of $\boldsymbol{B}$. Then for any $\varepsilon>0$, with probability approaching 1 as $n \rightarrow \infty$,

$$
\rho(B) \leq \sqrt{d}(1+\varepsilon) .
$$

These two lemmas imply Theorem B.1 via the approximations $A_{c, u} \approx \frac{u}{1-u^{2}} A-\frac{u d}{n} 11^{\top}$ and $D_{c, u} \approx \frac{u^{2}}{1-u^{2}} D$. Verification of this implication and proofs of these lemmas are contained in the full version of this paper.

\section{LOWER BOUNDS}

We outline the proof of the lower bounds in Theorems 2.2, 2.4, 2.5, and 2.7. We begin by reviewing some definitions and facts on local weak convergence in Section C.1, describing the sense in which the graphs $\mathrm{G}(n, d / n)$ and $\mathrm{G}(n, a / n, b / n)$ converge locally to GaltonWatson trees. We then reduce the lower bounds to the construction and analysis of local algorithms on such trees in Section C.2.

\section{C.1 Local Weak Convergence: Definitions}

To accommodate notationally both the Erdős-Rényi model and the stochastic-block-model, let $\mathcal{M}$ denote a general finite set of possible vertex labels. In the Erdős-Rényi case, we will simply take the trivial set $\mathcal{M}=\{1\}$; for the stochastic-block-model with partially revealed labels, we will take $\mathcal{M}=\{+1,-1, \mathrm{U}\}$ as described in Section 2. Then Definitions 2.1 and 2.6 coincide.

Let $\mathcal{G}(\mathcal{M})$ denote the space of tuples $(G,, \sigma)$ where $G=(V, E)$ is a (finite or) locally-finite graph, $\in V$ is a distinguished root vertex, and $\sigma: V \rightarrow \mathcal{M}$ associates a label to each vertex. Define a corresponding edge-perspective set $\mathcal{G}_{e}(\mathcal{M})$ as the space of tuples $\left(G,\left\{,^{\prime}\right\}, \sigma\right)$ where $G=(V, E)$ is a (finite or) locally-finite graph, $\sigma: V \rightarrow \mathcal{M}$ associates a label to each vertex, and $\left\{,^{\prime}\right\} \in E$ is a distinguished (undirected) root edge. The subspaces of trees in $\mathcal{G}(\mathcal{M}), \mathcal{G}_{e}(\mathcal{M})$ are denoted by $\mathcal{T}(\mathcal{M}), \mathcal{T}_{e}(\mathcal{M})$.
For any graph $H$, integer $\ell \geq 0$, and set of vertices $S$ in $H$, let $\mathrm{B}_{\ell}(S ; H)$ denote the subgraph induced by all vertices at distance at most $\ell$ from $S$ in $H$ (including $S$ itself). To make contact with previously introduced notation, for a vertex $v$, we write $\mathrm{B}_{\ell}(v ; H)$ for $\mathrm{B}_{\ell}(\{v\} ; H)$.

Local weak convergence was initially introduced in [BS01] and further developed in [AL07, AS04]. We define it here in a somewhat restricted setting that is relevant for our proofs.

Definition C.1. Let $v$ be a distribution over $\mathcal{T}(\mathcal{M})$ and let $\left\{G_{n}=\right.$ $\left.\left(V_{n}, E_{n}\right)\right\}_{n \geq 1}$ be a sequence of (deterministic) graphs with (deterministic) vertex labels $\sigma_{n}: V_{n} \rightarrow \mathcal{M}$. We say that $\left(G_{n}, \sigma_{n}\right)$ converges locally to $v$ if, for any $\ell \geq 0$, any $\tau \in \mathcal{T}(\mathcal{M})$, and a vertex $v \in V_{n}$ chosen uniformly at random ${ }^{5}$,

$$
\begin{aligned}
& \lim _{n \rightarrow \infty} \mathbb{P}\left\{\left(\mathrm{B}_{\ell}\left(v ; G_{n}\right), v, \sigma_{n}\right) \simeq \tau\right\} \\
& =\mathbb{P}_{v}\left\{\left(\mathrm{~B}_{\ell}(; T), \sigma\right) \simeq \tau\right\},
\end{aligned}
$$

where $\simeq$ denotes graph isomorphism that preserves the root vertex and vertex labels. We write $\left(G_{n}, \sigma_{n}\right) \Rightarrow v$.

A law $v$ over $\mathcal{T}(\mathcal{M})$ is the limit of some graph sequence if and only if $v$ is unimodular [BS01, Ele10, BLS15]. Roughly speaking, this means that the law $v$ does not change if the root is changed. Corresponding to any unimodular law $v$ is an associated edgeperspective law $v_{e}$ over $\mathcal{T}_{e}(\mathcal{M})$. This is obtained from $v$ as follows. First define a law $\tilde{v}$ over $\mathcal{T}(\mathcal{M})$ whose Radon-Nykodym derivative with respect to $v$ is $\frac{\mathrm{d} \tilde{v}}{\mathrm{~d} v}(T, v, \sigma)=\operatorname{deg}_{T}() / \mathbb{E}_{v} \operatorname{deg}_{T}()$. Then, letting $(T,, \sigma) \sim \tilde{v}$, define $v_{\boldsymbol{e}}$ to be the law of $(T,\{, v\}, \sigma)$ where $v$ is a uniformly random neighbor of in $T$.

The above definition is clarified by the following fact. Its proof is an immediate consequence of the definitions, once we notice that, in order to sample a uniformly random edge in $G_{n}$, it is sufficient to sample a random vertex $v$ with probability proportional to $\operatorname{deg}(v)$, and then sample one of its neighbors uniformly at random.

Lemma C.2. For $v$ a unimodular law over $\mathcal{T}(\mathcal{M})$, denote by $v_{e}$ the corresponding edge-perspective law. Let $\left\{G_{n}=\left(V_{n}, E_{n}\right)\right\}_{n \geq 1}$ be a graph sequence with vertex labels $\sigma_{n}: V_{n} \rightarrow \mathcal{M}$ such that $\left(G_{n}, \sigma_{n}\right) \Rightarrow v$. Then for any $\ell \geq 0$ and any $\tau_{e} \in \mathcal{T}_{e}(\mathcal{M})$, if an edge $\{u, v\} \in E_{n}$ is chosen uniformly at random, we have

$$
\begin{aligned}
& \lim _{n \rightarrow \infty} \mathbb{P}\left\{\left(\mathrm{B}_{\ell}\left(\{u, v\} ; G_{n}\right),\{u, v\}, \sigma_{n}\right) \simeq \tau_{e}\right\} \\
& =\mathbb{P}_{v_{e}}\left\{\left(\mathrm{~B}_{\ell}\left(\left\{,{ }^{\prime}\right\} ; T\right),\left\{{ }^{\prime}\right\}, \sigma\right) \simeq \tau_{e}\right\},
\end{aligned}
$$

where $\simeq$ denotes graph isomorphism that preserves the root edge and vertex labels.

Both the Erdős-Rényi random graph and the planted partition random graph with partially observed labels (revealed independently at random) satisfy the above definitions, where the laws $v$ and $v_{e}$ are the laws of Galton-Watson trees.

Definition C.3. A Galton-Watson tree with offspring distribution $\mu$ is a random tree rooted at a vertex, such that each vertex $v$ has $N_{v} \sim \mu$ children independently of the other vertices.

A two-type Galton-Watson tree with offspring distributions $\mu^{=}$and $\mu^{\neq}$is a random tree with binary vertex labels $\{+1,-1\}$ rooted

\footnotetext{
${ }^{5}$ Here $\sigma_{n}$ and $\sigma$ really denote the restrictions $\left.\sigma_{n}\right|_{\mathrm{B}_{\ell}\left(v ; G_{n}\right)}$ and $\left.\sigma\right|_{\mathrm{B}_{\ell}(; T)}$ of the labels to the balls of radius $\ell$. We avoid this type of cumbersome notation when the meaning is clear.
} 
at such that has label \pm 1 with equal probability, and each vertex has $N_{v}^{=} \sim \mu^{=}$children with same label as itself and $N_{v}^{\neq} \sim \mu^{\neq}$ children with opposite label from itself, independently of each other and of the other vertices.

The labels of a two-type Galton-Watson tree are partially revealed with probability $\delta$ if the set of labels is augmented to $\{+1,-1, U\}$ and, conditional on the tree, the label of each vertex is replaced by $\mathrm{U}$ independently with probability $1-\delta$.

Example C.4. Fix $d>0$. Let $G_{n}=\left(V_{n}, E_{n}\right) \sim \mathrm{G}(n, d / n)$ be an Erdős-Rényi random graph, let $\mathcal{M}=\{1\}$, and let $\sigma_{n} \equiv 1$ the trivial labeling. Then almost surely (over the realization of $G_{n}$ ), $\left(G_{n}, \sigma_{n}\right) \Rightarrow v$ where $v$ is the law of a Galton-Watson tree rooted at with offspring distribution Poisson $(d)$ (and labels $\sigma \equiv 1$ ).

The associated edge-perspective law $v_{e}$ is the law of two independent such trees rooted at and ' and connected by the single edge $\left\{,{ }^{\prime}\right\}$.

Example C.5. Fix $a, b>0$ and $\delta \in(0,1]$, let $G_{n}=\left(V_{n}, E_{n}\right) \sim$ $\mathrm{G}(n, a / n, b / n)$ be the planted partition random graph, and let $\sigma_{n}$ : $V_{n} \rightarrow\{+1,-1, \mathrm{U}\}$ be such that, independently for each vertex $i$, with probability $1-\delta$ we have $\sigma_{n}(i)=\mathrm{U}$, and with probability $\delta$ we have that $\sigma_{n}(i)$ equals the vertex label $(+1$ or -1$)$ of the hidden partition to which $i$ belongs. Then almost surely (over the realization of $G_{n}$ and $\left.\sigma_{n}\right),\left(G_{n}, \sigma_{n}\right) \Rightarrow v$ where $v$ is the law of a two-type Galton-Watson tree rooted at, with offspring distributions Poisson $(a / 2)$ and Poisson $(b / 2)$ and with vertices partially revealed with probability $\delta$.

The associated edge-perspective law $v_{e}$ is the law of two such trees rooted at and ' and connected by the single edge $\left\{,{ }^{\prime}\right\}$, where and ' belong to the same side of the partition with probability $a /(a+b)$ and to opposite sides of the partition with probability $b /(a+b)$, and the trees are independent conditional on the partition memberships of and'.

As in Section 2, to define local algorithms that solve the SDP (3), we extend our definitions to include additional random real-valued marks. Namely, we denote by $\mathcal{G}^{*}(\mathcal{M})$ the space of tuples $(G,, \sigma, z)$ where $(G,, \sigma) \in \mathcal{G}(\mathcal{M})$ and $z: V(G) \rightarrow \mathbb{R}$ associates a real-valued mark to each vertex of $G$. The spaces $\mathcal{G}_{e}^{*}(\mathcal{M}), \mathcal{T}^{*}(\mathcal{M}), \mathcal{T}_{e}^{*}(\mathcal{M})$ are defined analogously.

For a unimodular law $v$ over $\mathcal{T}(\mathcal{M})$, we let $v^{*}$ be the law over $\mathcal{T}^{*}(\mathcal{M})$ such that $(T,, \sigma, z) \sim v^{*}$ if $(T,, \sigma) \sim v$ and, conditional on $(T,, \sigma), z(i) \stackrel{i i d}{\sim} \operatorname{Normal}(0,1)$ for all vertices $i \in V(T) . v_{e}^{*}$ is defined analogously.

REMARK C.6. Since we are interested in graph sequences that converge locally to trees, it will turn out to be sufficient to define local algorithms $F: \mathcal{G}^{*}(\mathcal{M}) \rightarrow \mathbb{R}$ on trees and, for instance, extend it arbitrarily to other graphs. With a slight abuse of notation, we will therefore write $F: \mathcal{T}^{*}(\mathcal{M}) \rightarrow \mathbb{R}$.

Finally, given a local algorithm $F \in \mathcal{F}_{*}^{\mathcal{M}}(\ell)$ and a unimodular probability measure $v$ on $\mathcal{T}(\mathcal{M})$, we define the value of $F$ with respect to $v$ as

$$
\begin{aligned}
\mathcal{E}(F, v) & := \\
& d \mathbb{E}_{v_{e}^{*}}\left\{F\left(\mathrm{~B}_{\ell}(; T),, \sigma, z\right) F\left(\mathrm{~B}_{\ell}\left({ }^{\prime} ; T\right),{ }^{\prime}, \boldsymbol{\sigma}, \boldsymbol{z}\right)\right\},
\end{aligned}
$$

where $d=\mathbb{E}_{v}\{\operatorname{deg}()\}$ is the expected degree of the root under $v$. (This is a slight abuse of notation, given the definitions of $\mathcal{E}(F ; G)$ and $\mathcal{E}(F ; G, \sigma)$ in (4) and (14).)

\section{C.2 Construction and Analysis of Local Algorithms}

Using the above framework, the desired lower bounds are now consequences of the following results.

Lemma C.7. Let $\left\{G_{n}=\left(V_{n}, E_{n}\right)\right\}_{n \geq 1}$ be a deterministic sequence of graphs with deterministic vertex marks $\sigma_{n}: V_{n} \rightarrow \mathcal{M}$, such that $\left|V_{n}\right|=n,\left|E_{n}\right| / n \rightarrow d / 2$ for a constant $d>0$, and $\left(G_{n}, \sigma_{n}\right) \Rightarrow v$ for a law $v$ on $\mathcal{T}(\mathcal{M})$.

For fixed $\ell \geq 0$, let $F \in \mathcal{F}_{*}{ }^{\mathcal{M}}(\ell)$ be any radius- $\ell$ local algorithm such that the following two conditions hold ${ }^{6}$ :

$$
\begin{aligned}
\mathbb{E}_{v^{*}}\{F(T, \boldsymbol{\sigma}, \boldsymbol{z})\} & =0, \\
\mathbb{E}_{v^{*}}\left\{F(T, \boldsymbol{\sigma}, \boldsymbol{z})^{2} \mid T, \boldsymbol{\sigma}\right\} & \equiv 1 .
\end{aligned}
$$

Then we have

$$
\lim _{n \rightarrow \infty} \mathcal{E}\left(F ; G_{n}, \sigma_{n}\right) \geq \mathcal{E}(F, v) .
$$

A proof of the above lemma is provided in the full version of this paper.

Lemma C.8. Fix $d>1, \mathcal{M}=\{1\}$, and let $v$ be the law of the Galton-Watson tree with offspring distribution Poisson $(d)$ (and trivial marking $\sigma \equiv 1)$. Then there exist local algorithms $F_{\ell} \in \mathcal{F}_{*}^{\mathcal{M}}(\ell)$ for $\ell \geq 1$ satisfying (24) and such that

$$
\lim _{\ell \rightarrow \infty} \mathcal{E}\left(F_{\ell}, v\right) \geq 2 \sqrt{d}\left(1-\frac{1}{d+1}\right) .
$$

Proof SKeTCH. For any rooted tree $T$ and each vertex $v$ of $T$, let $k(v):=\operatorname{dist}(v$,$) denote the distance from v$ to the root . For each $\ell \geq 0$, denote

$$
N_{\ell}:=|\{v: k(v)=\ell\}|, \quad X_{\ell}:=d^{-\ell} N_{\ell} .
$$

Define the local algorithm

$$
F_{\ell}(T,, \sigma, z):= \begin{cases}\frac{\sum_{v \in \mathrm{B}_{\ell}(; T)} d^{-k(v) / 2} z(v)}{\sqrt{\sum_{v \in \mathrm{B}_{\ell}(; T)} d^{-k(v)}}} & X_{\ell}>0, \\ \operatorname{sign}\left(\sum_{v \in \mathrm{B}_{\ell}(; T)} z(v)\right) & X_{\ell}=0,\end{cases}
$$

where $k(v)$ and $X_{\ell}$ are defined for the tree $T$. When $z(v) \stackrel{\text { iid }}{\sim}$ $\operatorname{Normal}(0,1)$ conditional on $(T, \sigma)$, the conditions of $(24)$ hold by construction.

The proof may be completed by estimating $\mathcal{E}\left(F_{\ell}, v\right)$, using the martingale convergence of $X_{\ell}$ when $T$ is the Galton-Watson tree with offspring distribution Poisson $(d)$.

Lemma C.9. In the same setup as Lemma C.8, there exist local algorithms $F_{\ell, L} \in \mathcal{F}_{*}^{\mathcal{M}}(L)$ for $L \geq \ell \geq 1$ satisfying (24) and such that

$$
\lim _{\ell \rightarrow \infty} \lim _{L \rightarrow \infty} \mathcal{E}\left(F_{\ell, L}, v\right) \geq d \mathbb{E} \Psi\left(\mathrm{c}_{1}, \mathrm{c}_{2}\right),
$$

where $\Psi\left(\mathrm{c}_{1}, \mathrm{c}_{2}\right)$ is as in (9).

\footnotetext{
${ }^{6}$ The second of these is the same as condition 2 of Definition 2.6 and condition 2 of Definition 2.1 in the case of trivial markings $\mathcal{M}=\{1\}$; we restate it here for convenience.
} 
Proof Sketch. For a rooted tree $(T$,$) and any vertex v$ of $T$, let $k(v):=\operatorname{dist}(v$,$) denote the distance to the root. For any k>0$, let us call vertices $v$ for which $k(v)=k$ the 'leaf vertices' of the ball $\mathrm{B}_{k}(; T)$.

For $L \geq \ell \geq 1$, if $\mathrm{B}_{L}(; T)$ has at least one leaf vertex (i.e. $\{v$ : $k(v)=L\}$ is non-empty), then let us define a depth- $L$ approximation $h^{(, L)}(v ; T)$ to the harmonic measure introduced in Section 2.2, as follows: For any vertex $v \in \mathrm{B}_{L}(; T)$, let $k=k(v)$ and consider a simple random walk on $T$ starting at and ending when it visits the first leaf vertex of $\mathrm{B}_{L}(; T)$. Then let $h^{(, L)}(v ; T)$ be the probability that $v$ is the last vertex at distance $k$ from that is visited by this walk. Clearly, for each $k=0, \ldots, L$,

$$
\sum_{v \in T: k(v)=k} h^{(, L)}(v ; T)=1 .
$$

We may then define a local algorithm $F_{\ell, L} \in \mathcal{F}_{*}^{\mathcal{M}}(L)$ by

$$
\begin{aligned}
& F_{\ell, L}(T,, \sigma, z):= \\
& \begin{cases}\frac{\sum_{v \in \mathrm{B}_{\ell}(T)} \sqrt{h^{(, L)}(v ; T)} z(v)}{\sqrt{\ell+1}} & |\{v: k(v)=L\}|>0, \\
\operatorname{sign}\left(\sum_{v \in \mathrm{B}_{\ell}(; T)} z(v)\right) & \text { otherwise. }\end{cases}
\end{aligned}
$$

The conditions of (24) then hold by construction.

The proof may be completed by estimating $\mathcal{E}\left(F_{\ell, L}, v\right)$, using the relation between $h^{L}$ and the conductance c as $L \rightarrow \infty$.

Lemma C.10. Fix $a, b>0$ such that $d:=(a+b) / 2 \geq 2$ and $\lambda:=(a-b) / \sqrt{2(a+b)}>1$. Fix $\delta \in(0,1]$, let $\mathcal{M}=\{+1,-1, \mathrm{U}\}$, and let $v$ be the law of the two-type Galton-Watson tree with offspring distributions Poisson $(a / 2)$ and Poisson $(b / 2)$ and with vertex labels partially revealed with probability $\delta$. Then for a universal constant $C>0$, there exist local algorithms $F_{\ell} \in \mathcal{F}_{*}{ }^{\mathcal{M}}(\ell)$ for $\ell \geq 1$ satisfying (24) and such that

$$
\lim _{\ell \rightarrow \infty} \mathcal{E}\left(F_{\ell}, v\right) \geq \sqrt{d}\left(2+\frac{(\lambda-1)^{2}}{\lambda \sqrt{d}}-\frac{C}{d}\right) .
$$

Proof Sketch. Denote $\mu=(a-b) / 2$. For any rooted tree $(T,, \sigma)$ with vertex labels $\sigma: V(T) \rightarrow\{+1,-1, \mathrm{U}\}$, define by $k(v):=$ $\operatorname{dist}(v$,$) the distance from v$ to . For $\ell \geq 0$, define $X_{\ell}$ as in (27), and define

$$
\begin{aligned}
N_{\ell}^{+} & :=|\{v: k(v)=\ell, \sigma(v)=+1\}|, \\
N_{\ell}^{-} & :=|\{v: k(v)=\ell, \sigma(v)=-1\}|, \\
D_{\ell}: & =\delta^{-1} \mu^{-\ell}\left(N_{\ell}^{+}-N_{\ell}^{-}\right) .
\end{aligned}
$$

Note that $\left(X_{\ell}, D_{\ell}\right)$ is computable from the observed labels in $\mathrm{B}_{\ell}(; T)$. Define the local algorithm

$$
\begin{aligned}
& F_{\ell, \alpha}(T,, \sigma, z):= \\
& \begin{cases}\frac{\sum_{v \in \mathrm{B}_{\ell}(T)} d^{-k(v) / 2} z(v)+D_{\ell} \sqrt{\alpha \ell}}{\sqrt{\sum_{v \in \mathrm{B}_{\ell}(T)} d^{-k(v)}+D_{\ell}^{2} \alpha \ell}} & X_{\ell}>0, \\
\operatorname{sign}\left(\sum_{v \in \mathrm{B}_{\ell}(; T)} z(v)\right) & X_{\ell}=0,\end{cases}
\end{aligned}
$$

where $k(v)$ and $\left(X_{\ell}, D_{\ell}\right)$ are defined as above for the labeled tree $(T,, \sigma)$. (This perturbs the local algorithm constructed in Lemma C. 8 by a suitable multiple of $D_{\ell}$, whose consideration is loosely motivated by the asymptotic form of the second eigenvector of the non-backtracking matrix established in [BLM15].) The conditions of (24) hold by construction.
The proof is completed by estimating $\mathcal{E}\left(F_{\ell}, v\right)$, using tail bounds for the martingale $\left(X_{\ell}, D_{\ell}\right)$ when $T$ is a two-type Galton-Watson tree with offspring distributions Poisson $(a / 2)$ and Poisson $(b / 2)$.

Proof of Theorems 2.2, 2.4, 2.5, 2.7. The results (6), (10), and (15) follow from the above lemmas. Then (5), (8), and the second bound of (12) in the case $d \geq 2$ follow in turn, as any local algorithm defines a feasible solution $X$ for the SDP (3) which achieves the SDP value $n \mathcal{E}\left(F ; G_{n}, \sigma_{n}\right)$, as discussed in Section 2 . We may take $C>4$ so that the first bound dominates in (12) when $d \in(1,2)$.

For the first bound of (12), let us simply consider the feasible point $X=\sigma_{n} \sigma_{n}^{\top}$ for (3), where $\sigma_{n} \in\{+1,-1\}^{n}$ is the indicator vector of the hidden partition. Then

$$
\frac{1}{n} \operatorname{SDP}(\boldsymbol{A}) \geq \frac{1}{n}\left\langle\boldsymbol{A}-\frac{d}{n} \mathbf{1 1}^{\mathrm{\top}}, \boldsymbol{X}\right\rangle=\frac{1}{n} \sum_{i, j \in V_{n}} \boldsymbol{A}_{i j} \sigma_{n}(i) \sigma_{n}(j) .
$$

From the definition of $\mathrm{G}(n, a / n, b / n)$, we obtain almost surely

$$
\liminf _{n \rightarrow \infty} \frac{1}{n} \operatorname{SDP}(A) \geq \frac{1}{n}\left(\frac{n^{2}}{2} \cdot \frac{a}{n}-\frac{n^{2}}{2} \cdot \frac{b}{n}\right)=\lambda \sqrt{d} .
$$

The large $d$ expansion (11) in Theorem 2.4 is proven in the full version of this paper.

\section{REFERENCES}

$\left[\mathrm{ABC}^{+} 15\right]$ Pranjal Awasthi, Afonso S Bandeira, Moses Charikar, Ravishankar Krishnaswamy, Soledad Villar, and Rachel Ward. Relax, no need to round: Integrality of clustering formulations. In Proceedings of the 2015 Conference on Innovations in Theoretical Computer Science, pages 191-200. ACM, 2015.

[ABH16] Emmanuel Abbe, Afonso S Bandeira, and Georgina Hall. Exact recovery in the stochastic block model. IEEE Transactions on Information Theory, 62(1):471-487, 2016

[AL07] David Aldous and Russell Lyons. Processes on unimodular random networks. Electron. F. Probab, 12(54):1454-1508, 2007.

[AS04] David Aldous and J Michael Steele. The objective method: Probabilistic combinatorial optimization and local weak convergence. In Probability on discrete structures, pages 1-72. Springer, 2004.

[AV11] Brendan PW Ames and Stephen A Vavasis. Nuclear norm minimization for the planted clique and biclique problems. Mathematical programming, 129(1):69-89, 2011.

[Bas92] Hyman Bass. The Ihara-Selberg zeta function of a tree lattice. International fournal of Mathematics, 3(06):717-797, 1992.

[BCSZ14] Afonso S Bandeira, Moses Charikar, Amit Singer, and Andy Zhu. Multireference alignment using semidefinite programming. In Proceedings of the 5th conference on Innovations in theoretical computer science, pages 459-470. ACM, 2014

$\left[\mathrm{BHK}^{+} 16\right]$ Boaz Barak, Samuel B Hopkins, Jonathan Kelner, Pravesh K Kothari, Ankur Moitra, and Aaron Potechin. A nearly tight sum-of-squares lower bound for the planted clique problem. arXiv:1604.03084, 2016.

[BLM15] Charles Bordenave, Marc Lelarge, and Laurent Massoulié. Nonbacktracking spectrum of random graphs: community detection and non-regular Ramanujan graphs. In Foundations of Computer Science (FOCS), 2015 IEEE 56th Annual Symposium on, pages 1347-1357. IEEE, 2015.

[BLS15] Itai Benjamini, Russell Lyons, and Oded Schramm. Unimodular random trees. Ergodic Theory and Dynamical Systems, 35(02):359-373, 2015.

[BM03] Samuel Burer and Renato DC Monteiro. A nonlinear programming algorithm for solving semidefinite programs via low-rank factorization. Mathematical Programming, 95(2):329-357, 2003.

[BS01] Itai Benjamini and Oded Schramm. Recurrence of distributional limits of finite planar graphs. Electron. 7. Probab., 6:13 pp., 2001.

[CGHV15] Endre Csóka, Balázs Gerencsér, Viktor Harangi, and Bálint Virág. Invariant gaussian processes and independent sets on regular graphs of large girth. Random Structures \& Algorithms, 47:284-303, 2015.

[DKMZ11] Aurelien Decelle, Florent Krzakala, Cristopher Moore, and Lenka Zdeborová. Asymptotic analysis of the stochastic block model for modular networks and its algorithmic applications. Physical Review E, 84(6):066106, 2011. 
[DMS15] Amir Dembo, Andrea Montanari, and Subhabrata Sen. Extremal cuts of sparse random graphs. The Annals of Probability, 45(2):1190-1217, 2017.

[DM15a] Yash Deshpande and Andrea Montanari. Finding hidden cliques of size $\sqrt{N / e}$ in nearly linear time. Foundations of Computational Mathematics, 15(4):1069-1128, 2015.

[DM15b] Yash Deshpande and Andrea Montanari. Improved sum-of-squares lower bounds for hidden clique and hidden submatrix problems. In Proceedings of The 28th Conference on Learning Theory, pages 523-562, 2015.

[Ele10] Gábor Elek. On the limit of large girth graph sequences. Combinatorica, 30(5):553-563, 2010.

[FK00] Uriel Feige and Robert Krauthgamer. Finding and certifying a large hidden clique in a semirandom graph. Random Structures and Algorithms, 16(2):195-208, 2000.

[GS14] David Gamarnik and Madhu Sudan. Limits of local algorithms over sparse random graphs. In Proceedings of the 5th conference on Innovations in theoretical computer science, pages 369-376. ACM, 2014.

[GV15] Olivier Guédon and Roman Vershynin. Community detection in sparse networks via grothendieckâĂŹs inequality. Probability Theory and Related Fields, pages 1-25, 2015

[GW95] Michel X Goemans and David P Williamson. Improved approximation algorithms for maximum cut and satisfiability problems using semidefinite programming. Fournal of the ACM (FACM), 42(6):1115-1145, 1995.

[HLS14] Hamed Hatami, László Lovász, and Balázs Szegedy. Limits of locallyglobally convergent graph sequences. Geometric and Functional Analysis, 24(1):269-296, 2014.

[HWX15] Bruce Hajek, Yihong Wu, and Jiaming Xu. Achieving exact cluster recovery threshold via semidefinite programming: Extensions. arXiv: $1502.07738,2015$

[HWX16] Bruce Hajek, Yihong Wu, and Jiaming Xu. Achieving exact cluster recovery threshold via semidefinite programming. IEEE Transactions on Information Theory, 62(5):2788-2797, 2016.

[Jer92] Mark Jerrum. Large cliques elude the metropolis process. Random Structures \& Algorithms, 3(4):347-359, 1992.

[JLR11] Svante Janson, Tomasz Luczak, and Andrzej Rucinski. Random graphs, volume 45. John Wiley \& Sons, 2011

[JMRT16] Adel Javanmard, Andrea Montanari, and Federico Ricci-Tersenghi. Phase transitions in semidefinite relaxations. Proceedings of the National Academy of Sciences, 113(16):E2218-E2223, 2016.

$\mathrm{KMM}^{+}$13] Florent Krzakala, Cristopher Moore, Elchanan Mossel, Joe Neeman, Allan Sly, Lenka Zdeborová, and Pan Zhang. Spectral redemption in cluster ing sparse networks. Proceedings of the National Academy of Sciences, 110(52):20935-20940, 2013.

[KS00] Motoko Kotani and Toshikazu Sunada. Zeta functions of finite graphs. F. Math. Sci. Univ. Tokyo, 7:7-25, 2000.
[LPP95] Russell Lyons, Robin Pemantle, and Yuval Peres. Ergodic theory on galtonâĂTwwatson trees: Speed of random walk and dimension of harmonic measure. Ergodic Theory and Dynamical Systems, 15(03):593-619, 1995.

[Lyo14] Russell Lyons. Factors of iid on trees. arXiv:1401.4197, 2014.

[Mas14] Laurent Massoulié. Community detection thresholds and the weak Ramanujan property. In Proceedings of the 46th Annual ACM Symposium on Theory of Computing, pages 694-703. ACM, 2014.

[MPW16] Ankur Moitra, William Perry, and Alexander S. Wein. How robust are reconstruction thresholds for community detection? In Proceedings of the 48th Annual ACM Symposium on Theory of Computing, pages 828-841. ACM, 2016.

[MNS12] Elchanan Mossel, Joe Neeman, and Allan Sly. Stochastic block models and reconstruction. arXiv:1202.1499, 2012

[MNS13] Elchanan Mossel, Joe Neeman, and Allan Sly. A proof of the block model threshold conjecture. arXiv:1311.4115, 2013.

[Mon15] Andrea Montanari. Finding one community in a sparse graph. Journal of Statistical Physics, 161(2):273-299, 2015.

[MPW15] Raghu Meka, Aaron Potechin, and Avi Wigderson. Sum-of-squares lowe bounds for planted clique. In Proceedings of the Forty-Seventh Annual ACM on Symposium on Theory of Computing, pages 87-96. ACM, 2015.

MS16] Andrea Montanari and Subhabrata Sen. Semidefinite programs on sparse random graphs and their application to community detection. In Proceedings of the 48th Annual ACM SIGACT Symposium on Theory of Computing, pages 814-827. ACM, 2016

[MX16] Elchanan Mossel and Jiaming Xu. Local algorithms for block models with side information. In Proceedings of the 2016 ACM Conference on Innovations in Theoretical Computer Science, pages 71-80. ACM, 2016.

[NN13] Yurii Nesterov and Arkadi Nemirovski. On first-order algorithms for 1 1/nuclear norm minimization. Acta Numerica, 22:509-575, 2013.

[RV14] Mustazee Rahman and Balint Virag. Local algorithms for independent sets are half-optimal. arXiv:1402.0485, 2014.

[SKZ14] Alaa Saade, Florent Krzakala, and Lenka Zdeborová. Spectral clustering of graphs with the Bethe Hessian. In Advances in Neural Information Processing Systems, pages 406-414, 2014

[Suo13] Jukka Suomela. Survey of local algorithms. ACM Computing Surveys (CSUR), 45(2):24, 2013

[WS08] Kilian Q Weinberger and Lawrence K Saul. Fast solvers and efficient implementations for distance metric learning. In Proceedings of the 25th international conference on Machine learning, pages 1160-1167. ACM, 2008.

[ZMZ14] Pan Zhang, Cristopher Moore, and Lenka Zdeborová. Phase transitions in semisupervised clustering of sparse networks. Physical Review E, 90(5):052802, 2014 